CLINICAL RESEARCH

\title{
Predictive value of myenteric and submucosal plexitis for postoperative Crohn's disease recurrence
}

\author{
Sayumi Nakao", Michio Itabashi' ${ }^{1)}$, Tomoko Yamamoto ${ }^{2)}$ and Takahiro Okamoto ${ }^{1)}$ \\ 1) Department of Surgery 2, Tokyo Women's Medical University \\ 2) Department of Surgical Pathology, Tokyo Women's Medical University
}

\begin{abstract}
:
Objectives: To assess the predictive values of myenteric and submucosal plexitis for postoperative endoscopic recurrence of Crohn's disease (CD). Methods: A retrospective study of CD patients who underwent intestinal resection between 1995 and 2013 in the Department of Surgery 2, Tokyo Women's Medical University was performed. Proximal resection margins were analyzed and plexitis was evaluated by counting the number of inflammatory cells in myenteric and submucosal plexuses. The sizes of the most severely inflamed ganglion (MIG) were measured. Multiple regression analysis was used to identify independent risk factors for postoperative endoscopic recurrence. Results: Of the 51 included patients, 40 patients underwent colonoscopy after surgery. Endoscopic recurrence was observed in 21 patients (52.5\%). Mean duration ( \pm standard deviation) from surgery to recurrence was $49.7 \pm 34.7$ months. Endoscopic recurrence rates at 1 , 3 , and 5 years were $5.0 \%, 24.1 \%$, and $45.1 \%$, respectively. Submucosal plexitis and myenteric plexitis were observed in $36(90.0 \%)$ and 37 patients $(92.5 \%)$, respectively. On multivariate analysis, initial intestinal resection, rate of plexitis $<50 \%$, size of the MIG in the myenteric plexus $\geq 867 \mu \mathrm{m}^{2}$, and total number of inflammatory cells in the submucosal plexus $\geq 8$ were independent risk factors for postoperative endoscopic recurrence. Conclusions: Pathological findings of proximal resection margins, especially submucosal plexitis and large sizes of myenteric plexus, are predictive of postoperative endoscopic recurrence in CD.
\end{abstract}

Keywords:

Crohn's disease, postoperative recurrence, risk factor, submucosal plexitis, myenteric plexitis

J Anus Rectum Colon 2017; 1(2): 56-64

\section{Introduction}

Crohn's disease (CD) is an inflammatory disease of unknown etiology that can affect any segment of the gastrointestinal tract ${ }^{1}$. Even in the era of biologics (infliximab and adalimumab), surgery is still needed within 5 years after diagnosis in $25 \%$ to $33 \%$ of patients ${ }^{2}$. However, surgery is not curative and postoperative recurrence is still a major problem. Recurrence can be defined in several ways depending on the diagnostic modality used, with clinical, endoscopic, and surgical recurrences being most commonly reported. Among these, endoscopic recurrence occurs earlier than the other types of recurrence ${ }^{3)}$, occurring in approximately $22 \%$, $56 \%$, and $74 \%$ of patients by 1,5 , and 10 years after surgery, respectively ${ }^{4}$. Common relapse sites for $\mathrm{CD}$ lesions are the anastomosis and neoterminal ileum ${ }^{5,6}$.

Several risk factors have been identified for postoperative recurrence of CD. Smoking, history of previous intestinal resection, penetrating disease, and perianal disease are the commonly reported clinical risk factors ${ }^{7-9)}$. Similarly, several histological factors have been studied to identify risk factors for postoperative recurrence. However, the predictive abilities of most of the histological factors, including granulomas and pathological involvement of resection margins, remain controversial ${ }^{5,10-12)}$.

In $\mathrm{CD}$ patients, inflammatory infiltrates associated with both the submucosal and myenteric nerve plexuses have been observed ${ }^{133}$. In 2006, Ferrante et al. ${ }^{14)}$ first identified the presence of myenteric plexitis in the proximal resection margin as a predictor of postoperative endoscopic recurrence of 
CD. Several studies to date have proposed the possibility of myenteric or submucosal plexitis as a predictor of postoperative recurrence ${ }^{15-18)}$. However, the definition of plexitis and the optimal methods for evaluation have yet to be firmly established. The aims of the current study were to reveal the occurrence rate of plexitis and to assess the predictive value of myenteric and submucosal plexitis in the proximal resection margin of the intestine for postoperative endoscopic recurrence of CD.

\section{Methods}

\section{Patients}

A retrospective study of a single-center cohort was performed. Between January 1995 and March 2013, a total of 240 patients underwent surgery for intestinal lesions of CD in the Department of Surgery 2 at Tokyo Women's Medical University, Japan. Patients with ileocolonic or colonic resection and ileocolonic or colo-colonic anastomosis, and who were over 20 years of age at the time of the study, were included in this study. Exclusion criteria were absence of a labeled proximal margin and unavailability for postoperative follow-up for more than 2 years. Fifty-one patients met all the inclusion and exclusion criteria. This study was approved by the ethics review board of Tokyo Women's Medical University (Ethical Committee Approval No. 3549-R). All study participants provided written informed consent prior to study enrollment.

\section{Clinical data collection}

For each patient, the following data were extracted from medical charts: demographic information, clinical setting of CD (age at onset, disease duration, Montreal classification ${ }^{19)}$, etc.), past medical history, and smoking status. Surgeryrelated data were also recorded, including age at surgery, pre- and postoperative treatments, surgical indications, type of surgery, type of anastomosis, presence or absence of residual lesions, and postoperative complications.

\section{Postoperative recurrence}

Surgical recurrence was defined as recurrence at the anastomotic site necessitating an operation. Endoscopic recurrence was scored according to Rutgeerts' endoscopic scoring system $^{20)}$. Significant endoscopic recurrence was defined as Rutgeerts' score of i2 or higher confined to the anastomotic site (within 2.5 centimeters from the anastomosis to both oral and anal side).

\section{Pathological examination}

The proximal resection margins of 46 ileocolonic resections and 5 colonic resections were analyzed by one expert pathologist (T.Y.) and the first author (S.N.), both of whom were blinded to the postoperative outcomes of the patients.

Each sample was fixed in $20 \%$ formalin and analyzed using hematoxylin and eosin (H\&E) stain. Immunochemistry of serial sections was used to detect T-lymphocytes (antiCD3 antibody) and mastocytes (anti-CD117 antibody). Sections were treated with $3 \% \mathrm{H}_{2} \mathrm{O}_{2}$ in Phosphate buffered saline (PBS) for $10 \mathrm{~min}$ to block endogenous peroxidase, and they were transferred to $5 \%$ skim milk at room temperature (RT) for $10 \mathrm{~min}$. The tissue was incubated with anti-CD3 (M7254, mouse, 1:1,000, Dako, Glostrup, Denmark) and anti-CD117 (A4502, rabbit, 1:1,000, Dako) antibodies, overnight at $4^{\circ} \mathrm{C}$. Microwave antigen retrieval with Trisethylenediaminetetraacetic acid (EDTA) ( $\mathrm{pH}$ 9.0) was performed for CD3. After washing, the tissue was treated with a solution of EnVision kit/HRP (K5027, Dako) at RT for 30 min. The color was developed with the chromogen diaminobenzidine tetrahydrochloride, and the slides were counterstained with hematoxylin.

Each resection specimen was assessed for the typical lesions of $\mathrm{CD}$, including inflammatory infiltrates, erosions, crypt abscesses, and granulomas. Special attention was paid to the enteric nervous system. The myenteric and submucosal plexuses were assessed independently. The number of ganglia (defined as an accumulation of neuronal cell bodies and Schwann cells, with at least two neural cell bodies) within five millimeters from the proximal margin was counted. Adjacent ganglia without intervening structures such as blood vessels were counted as one plexus. Plexitis was defined as the presence of one or more inflammatory cells contiguous with or within an enteric ganglion. Plexitis was evaluated based on the appearance of the most severely inflamed ganglion (MIG). The number of inflammatory cells (mononuclear cells and polymorphonuclear leukocytes) contiguous with or within each ganglion was counted, and one ganglion in which the number of inflammatory cells was the largest was selected. The severity of plexitis in the MIG was graded according to the classification system proposed by Ferrante et al. as mild (G1) if the MIG contained <4 inflammatory cells, moderate (G2) if it contained 4-9 cells, or severe (G3) if it contained $\geq 10$ cells $^{14}$. Dimensions of the MIG were measured using a digital photomicrographic camera (DP26; Olympus Corporation, Tokyo, Japan). The MIG of each resection specimen was also assessed using both CD3stained and CD117-stained slides to count the numbers of Tlymphocytes and mastocytes, respectively. Some examples of each staining are shown in Figure 1.

\section{Statistical analysis}

JMP Pro11 (SAS Institute, Cary, NC, USA) was used for all statistical analyzes. All quantitative variables are reported as means ( \pm standard deviation). Cumulative probabilities of surgical and endoscopic recurrence-free survival were estimated using the Kaplan-Meier method. Univariate analysis (non-parametric Wilcoxon two-sample test for continuous variables and chi-squared test for categorical variables) was performed to look for possible predictors of postoperative endoscopic recurrence. When considering continuous variables for dichotomous analysis, cut-off values were determined using receiver operating characteristic (ROC) curve 


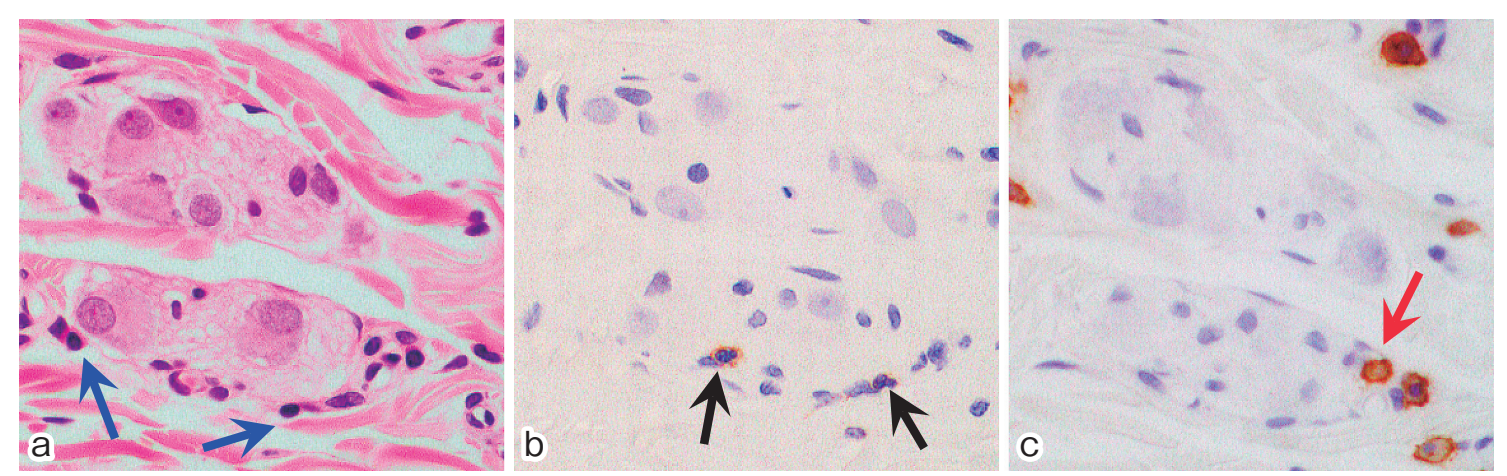

Figure 1. Pathological assessment of the intestinal plexus.

a: Submucosal plexitis with mononuclear cells contiguous to the ganglion (blue arrows). (H\&E staining, 400x).

b: Submucosal plexitis with T-cell lymphocytes within and contiguous with the ganglion (black arrows). (immunostaining with anti-CD3 antibody, 400x).

c: Submucosal plexitis with a mastocyte contiguous with the ganglion (red arrow). (immunostaining with antiCD117 antibody, 400x).

analysis, using the outcome event as a classification variable. To identify independent risk factors for postoperative endoscopic recurrence using multivariate analysis, all significant variables evaluated on univariate analysis were integrated into multiple logistic regression. The logistic model was assessed with R-squared, an index of contribution to the model, and area under the receiver operating characteristic curve. Values of $P<0.05$ were considered significant.

\section{Results}

\section{Baseline characteristics of the patients}

The baseline characteristics of the 51 patients (38: male) are shown in Table 1A. The mean age at diagnosis was 24.9 \pm 9.7 years and the mean disease duration was $113.3 \pm 92.0$ months. Eleven $(21.5 \%)$ patients had perianal disease and 13 $(25.4 \%)$ were active smokers. The majority of patients were on medical therapy at the time of surgery.

\section{Postoperative recurrence}

Data for postoperative evaluation of the anastomotic site by colonoscopy were available from 40 patients (78.4\%). The mean duration from surgery to endoscopic investigation was 33.6 \pm 31.9 months. Endoscopic recurrence (Rutgeerts score $\geq$ i2) was observed in 21 patients $(52.5 \%)$. The mean interval between surgery and endoscopic recurrence was $49.7 \pm 34.7$ months. Endoscopic recurrence rates at 1, 3, and 5 years were $5.0 \%, 24.1 \%$, and $45.1 \%$, respectively. Surgical recurrence was observed in four patients $(10.0 \%)$. The mean interval between surgery and surgical recurrence was $67.7 \pm$ 41.0 months. Surgical recurrence rates at 1,3 , and 5 years were $0.0 \%, 2.5 \%$, and $8.4 \%$, respectively.

\section{Pathological findings}

The pathological features of the 51 patients are summarized in Table 1B. Granulomas were present in 5 patients (9.8\%). Submucosal plexitis was observed in 45 patients
(88.2\%), while myenteric plexitis was observed in 47 patients $(92.2 \%)$.

\section{Correlation between severity of plexitis and postoperative endoscopic recurrence}

The severity of plexitis in the MIG was identified by pathological examination of resected specimens in the 40 patients who underwent colonoscopy after surgery. Submucosal plexitis was absent (G0), G1, G2, and G3 in 4, 23, 11, and 2. Endoscopic recurrence was observed in $2(50.0 \%), 11$ $(47.8 \%), 6(54.5 \%)$, and $2(100.0 \%)$ in each grade. Myenteric plexitis was absent (G0), G1, G2, and G3 in 3, 19, 15, and 3. Endoscopic recurrence was observed in 1 (33.3\%), 10 $(52.6 \%), 7(46.7 \%)$, and $3(100.0 \%)$ in each grade. Endoscopic recurrence rates in each grade were almost the same in both submucosal plexus $(\mathrm{p}=0.564)$ and myenteric plexus $(\mathrm{p}=0.339)$, except in $\mathrm{G} 3$, which showed recurrence rates of $100 \%$ for both submucosal and myenteric plexus. G3 plexitis was observed only in patients with endoscopic recurrence in both submucosal and myenteric plexuses.

\section{Risk factors for postoperative endoscopic recurrence}

Univariate analysis of the risk factors for postoperative endoscopic recurrence was performed in 40 patients and the variables tested are shown in Table $2 \mathrm{~A}$ and 3A. Surgery performed before the year $2004(\mathrm{p}=0.044)$, initial intestinal resection $(\mathrm{p}=0.011)$, rate of plexitis (number of ganglia with plexitis/number of ganglia analyzed $)<50 \%(\mathrm{p}=0.031)$, size of the MIG in the myenteric plexus $\geq 867 \mu \mathrm{m}^{2} \quad(\mathrm{p}=0.009)$, and total number of inflammatory cells in the submucosal plexus $\geq 8 \quad(p=0.044)$ were associated with an increased risk of endoscopic recurrence. On multivariate analysis, initial intestinal resection, rate of plexitis $<50 \%$, size of the MIG in the myenteric plexus $\geq 867 \mu \mathrm{m}^{2}$, and total number of inflammatory cells in the submucosal plexus $\geq 8$ were independently associated with endoscopic recurrence. R-squared of the logistic model was 0.598 and the area under the ROC curve was 0.934 . 
Table 1. Baseline Characteristics and Pathological Findings of the 51 Included Patients.

\begin{tabular}{|c|c|c|c|c|}
\hline & \multicolumn{2}{|c|}{$\mathrm{n}(\%)$} & Mean count & \\
\hline \multicolumn{5}{|l|}{ (A) Baseline characteristics } \\
\hline \multicolumn{3}{|l|}{ Age at the time of surgery $(\mathrm{Y})$} & \multicolumn{2}{|l|}{$34.2 \pm 10.7$} \\
\hline \multicolumn{3}{|l|}{ Disease duration $(\mathrm{M})$} & \multicolumn{2}{|l|}{$113.3 \pm 92.0$} \\
\hline \multicolumn{5}{|l|}{ Montreal classification } \\
\hline Disease location L1/L2/L3/L4 & \multicolumn{4}{|c|}{$9(17.6) / 3(5.9) / 39(76.5) / 0(0)$} \\
\hline Disease behavior B1/B2/B3 & \multicolumn{4}{|c|}{$2(3.9) / 26(51.0) / 23(45.1)$} \\
\hline \multicolumn{5}{|l|}{ Preoperative treatment } \\
\hline 5-ASA/Steroids/IM/Anti-TNF $\alpha /$ Exclusive parenteral nutrition & \multicolumn{4}{|c|}{$\begin{array}{l}40(78.4) / 21(41.2) / 13(25.4) / \\
7(13.7) / 28(54.9)\end{array}$} \\
\hline Previous intestinal resection & \multicolumn{4}{|c|}{$23(45.0)$} \\
\hline Type of surgery (Ileocolonic/Colonic resection) & \multicolumn{4}{|c|}{$46(90.2) / 5(9.8)$} \\
\hline Open/Laparoscopic surgery & \multicolumn{4}{|c|}{$30(58.8) / 21(41.2)$} \\
\hline Emergency operation & \multicolumn{4}{|c|}{$4(7.8)$} \\
\hline Anastomosis (Stapled/ Hand-sewn) * & \multicolumn{4}{|c|}{$41 / 49(83.6) / 8 / 49(16.3)$} \\
\hline Presence of residual lesion** & \multicolumn{4}{|c|}{$18 / 48(37.5)$} \\
\hline Postoperative complications $^{+}$ & \multicolumn{4}{|l|}{$14(27.4)$} \\
\hline \multicolumn{5}{|l|}{ Postoperative treatment } \\
\hline IM/Anti-TNF $\alpha /$ Therapy intensification & \multicolumn{4}{|c|}{$15(29.4) / 24(47.0) / 22(43.1)$} \\
\hline \multicolumn{5}{|c|}{ (B) Pathological findings } \\
\hline \multirow[t]{3}{*}{ Granulomas/Erosions/Crypt abscess/Inflammatory infiltration of mucosa } & \multicolumn{4}{|c|}{$5(9.8) / 1(1.9) / 1(1.9) / 6(11.7)$} \\
\hline & \multicolumn{2}{|c|}{ 1) Submucosal plexus } & \multicolumn{2}{|c|}{ 2) Myenteric plexus } \\
\hline & $\mathrm{n}(\%)$ & Mean count & $\mathrm{n}(\%)$ & Mean count \\
\hline Ganglia analyzed & & $9.4 \pm 6.7$ & & $5.8 \pm 3.0$ \\
\hline Ganglia with plexitis & & $5.5 \pm 5.3$ & & $3.4 \pm 2.3$ \\
\hline Plexitis & $45(88.2)$ & & $47(92.1)$ & \\
\hline Size of MIG $\left(\mu \mathrm{m}^{2}\right)$ & & $428.6 \pm 795.2$ & & $1,685.8 \pm 1,438.8$ \\
\hline \multicolumn{5}{|l|}{ Cellular infiltrates } \\
\hline Mononuclear cells & $43(84.3)$ & $3.5 \pm 7.1$ & $47(92.1)$ & $3.5 \pm 3.1$ \\
\hline Polymorphonuclear leukocytes & $10(19.6)$ & $0.7 \pm 2.7$ & $12(23.5)$ & $0.5 \pm 1.2$ \\
\hline T-lymphocytes* & $19(37.2)$ & $1.9 \pm 7.9$ & $33(64.7)$ & $2.1 \pm 2.4$ \\
\hline Mastocytes* & $29(56.8)$ & $1.2 \pm 2.3$ & $9(17.6)$ & $0.2 \pm 0.7$ \\
\hline \multicolumn{5}{|l|}{ 1)+2) } \\
\hline Total ganglia analyzed & & $15.3 \pm 8.3$ & & \\
\hline Total ganglia with plexitis & & $9.0 \pm 6.7$ & & \\
\hline
\end{tabular}

*Data were unavailable in 2 cases. **Data were unavailable in 3 cases.

*T-lymphocytes and mastocytes were detected by immunohistochemistry. All other items were examined on HE slides.

${ }^{+}$Clavien-Dindo classification $\geq 2$.

n: Number; Y: Years; M: Months; IM: Immunomodulators; TNF: Tumor necrosis factor; MIG: Most severely inflamed ganglion.

Therapy intensification: postoperative usage of immunomodulators or anti-TNF $\alpha$ in patients who were naïve to these drugs before surgery.

Next, to eliminate the confounding effects of a prolonged interval between surgery and endoscopic recurrence on the endoscopic recurrence rate, endoscopic recurrence was limited to within 3 years from surgery. Among the 40 patients studied, 28 patients underwent colonoscopy within 3 years from surgery. Endoscopic recurrence was observed in nine of these patients $(32.1 \%)$. The median interval between surgery and endoscopic recurrence was $20.6 \pm 9.8$ months. On univariate analysis, disease duration $\geq 57$ months $(\mathrm{p}=0.035$ ), number of submucosal ganglia with plexitis $\geq 19(\mathrm{p}=0.033)$, number of analyzed submucosal and myenteric ganglia $\geq 11$ ( $\mathrm{p}=0.035$ ), size of the MIG in the myenteric plexus $\geq 2,966$ $\mu \mathrm{m}^{2}(\mathrm{p}=0.007)$, number of polymorphonuclear leukocytes in the submucosal plexus $\geq 3$ ( $\mathrm{p}=0.007$ ), number of polymorphonuclear leukocytes in the myenteric plexus $\geq 5$ ( $\mathrm{p}=0.003$ ), and number of mastocytes in the myenteric plexus $\geq 1$ ( $\mathrm{p}=$ 0.01 ) were associated with an increased risk of endoscopic recurrence (Table 2B and 3B). Multivariate analysis did not identify any of these variables as significantly associated with short-term endoscopic recurrence.

\section{Dimensions and inflammatory infiltrates in the MIG}

In the preceding results, the size of the MIG in the myenteric plexus was an independent risk factor for endoscopic recurrence. However, the number of inflammatory infiltrates in the myenteric plexus was not a predictor of endoscopic recurrence. Conversely, in the submucosal plexus, the number of inflammatory infiltrates was an independent risk factor, whereas the size of the MIG was not. Therefore, the differences in dimensions and number of inflammatory infil- 
Table 2. Univariate Analysis of Clinical Risk Factors Associated with Postoperative Endoscopic Recurrence.

\begin{tabular}{|c|c|c|c|c|c|c|c|c|}
\hline \multirow{2}{*}{ Variable } & \multicolumn{4}{|c|}{ (A) Endoscopic recurrence } & \multicolumn{4}{|c|}{ (B) Endoscopic recurrence in $3 Y$} \\
\hline & Yes $(n=21)$ & No $(n=19)$ & Cut-off & $P$ & Yes $(n=21)$ & No $(n=19)$ & Cut-off & $P$ \\
\hline Male sex & $15(71.4)$ & $14(73.7)$ & & 0.873 & $5(55.6)$ & $12(63.2)$ & & 0.700 \\
\hline Age at diagnosis $(\mathrm{Y})$ & $8(38.1)$ & $12(63.2)$ & $\geq 23$ & 0.113 & $3(33.3)$ & $2(10.5)$ & $\geq 38$ & 0.141 \\
\hline Age at the time of surgery $(\mathrm{Y})$ & $6(28.6)$ & $10(52.6)$ & $\geq 37$ & 0.120 & $3(33.3)$ & $3(15.8)$ & $\geq 45$ & 0.290 \\
\hline Disease duration $(\mathrm{M})$ & $2(9.5)$ & $2(10.5)$ & $\geq 278$ & 0.915 & $8(88.9)$ & $9(47.4)$ & $\geq 57$ & 0.035 \\
\hline \multicolumn{9}{|l|}{ Montreal classification } \\
\hline L1 (vs. L2 \& L3) & $4(19.0)$ & $4(21.1)$ & & 0.874 & $1(11.1)$ & $5(26.3)$ & & 0.359 \\
\hline B3 (vs. B1 \& B2) & $9(42.9)$ & $8(57.1)$ & & 0.961 & $4(44.4)$ & $7(36.8)$ & & 0.700 \\
\hline Perianal disease & $2(9.5)$ & $4(21.1)$ & & 0.307 & $0(0)$ & $2(10.5)$ & & 0.312 \\
\hline Active smoking & $6(28.6)$ & $3(15.8)$ & & 0.333 & $2(22.2)$ & $2(10.5)$ & & 0.408 \\
\hline \multicolumn{9}{|l|}{ Preoperative treatment } \\
\hline 5-ASA & $17(81.0)$ & $15(78.9)$ & & 0.874 & $7(77.8)$ & $16(84.2)$ & & 0.678 \\
\hline Steroids & $11(52.4)$ & $6(31.6)$ & & 0.183 & $5(55.6)$ & $8(57.1)$ & & 0.505 \\
\hline IM & $5(23.8)$ & $5(26.3)$ & & 0.855 & $4(44.4)$ & $5(26.3)$ & & 0.337 \\
\hline Anti-TNF $\alpha$ & $2(9.5)$ & $3(15.8)$ & & 0.549 & $1(11.1)$ & $3(15.8)$ & & 0.741 \\
\hline Exclusive parenteral nutrition & $9(42.9)$ & $13(68.4)$ & & 0.104 & $4(44.4)$ & $14(73.7)$ & & 0.131 \\
\hline${ }^{+}$Preoperative anemia & $15(71.4)$ & $16(84.2)$ & & 0.333 & $5(55.6)$ & $15(78.9)$ & & 0.200 \\
\hline 'Preoperative low serum albumin level & $14(66.7)$ & $11(57.9)$ & & 0.567 & $6(66.7)$ & $10(52.6)$ & & 0.483 \\
\hline 'Preoperative high serum CRP level & $10(47.6)$ & $9(47.9)$ & & 0.987 & $4(44.4)$ & $10(52.6)$ & & 0.685 \\
\hline Year of operation (before 2004) & $8(38.1)$ & $2(10.5)$ & & 0.044 & $1(11.1)$ & $3(15.8)$ & & 0.741 \\
\hline Initial intestinal resection & $16(76.2)$ & $7(36.8)$ & & 0.011 & $6(66.7)$ & $10(52.6)$ & & 0.483 \\
\hline Emergency operation & $3(14.3)$ & $1(5.3)$ & & 0.342 & $1(11.1)$ & $2(10.5)$ & & 0.457 \\
\hline Laparoscopic surgery (vs. open) & $10(47.6)$ & $6(31.6)$ & & 0.301 & $4(44.4)$ & $8(42.1)$ & & 0.907 \\
\hline Stapled anastomosis (vs. hand-sewn) & $19(90.5)$ & $15(78.9)$ & & 0.505 & $8(88.9)$ & $15(78.9)$ & & 0.701 \\
\hline Presence of residual lesion & $7(33.3)$ & $4(21.1)$ & & 0.446 & $3(33.3)$ & $4(21.1)$ & & 0.468 \\
\hline Postoperative complications & $6(28.6)$ & $4(21.1)$ & & 0.583 & $2(22.2)$ & $6(31.6)$ & & 0.608 \\
\hline \multicolumn{9}{|l|}{ Postoperative treatment } \\
\hline $\mathrm{IM}$ & $5(23.8)$ & $7(36.8)$ & & 0.369 & $4(44.4)$ & $5(26.3)$ & & 0.337 \\
\hline Anti-TNF $\alpha$ & $11(52.4)$ & $8(57.1)$ & & 0.515 & $6(66.7)$ & $10(52.6)$ & & 0.483 \\
\hline Therapy intensification & $9(42.9)$ & $10(52.6)$ & & 0.536 & $6(66.7)$ & $10(52.6)$ & & 0.483 \\
\hline
\end{tabular}

$P$ values were calculated by chi-squared tests.

${ }^{+}$Based on the criterion value of the hospital.

Y: Years; M: Months; IM: Immunomodulators; TNF: Tumor necrosis factor; CRP: C-reactive protein.

trates per unit area of the MIG (number of inflammatory infiltrates in MIG/dimensions of MIG $\left.\left(\mu \mathrm{m}^{2}\right) \times 100\right)$ between the recurrence and non-recurrence groups were investigated. The results of the univariate analysis are shown in Table 4. The MIG in both the submucosal and myenteric plexuses tended to be larger in the recurrence group than in the nonrecurrence group, while the number of cellular infiltrates per unit area tended to be smaller in the recurrence group, although neither difference was significant.

\section{Discussion}

In $\mathrm{CD}$, postoperative recurrence is still a major problem, which may lead to significant morbidity and impaired quality of life. Even though the involved intestines are removed surgically, $50 \%$ to $70 \%$ of operated patients experience recurrence $^{4,5)}$. Furthermore, $30 \%-70 \%$ of the patients will require another intestinal resection ${ }^{2,3,21}$. A recent study confirmed that it is essential to choose postoperative treatments based on the individual risk of recurrence for the prevention of postoperative recurrence ${ }^{22)}$.

Inflammatory recurrence at the site of anastomosis, which can be observed endoscopically, appears earlier than clinical symptoms ${ }^{23)}$. This study therefore focused on endoscopic recurrence as one of the endpoints that could be evaluated objectively using Rutgeert's endoscopic scoring system ${ }^{20}$. Endoscopic recurrence rate of the present study was $52.5 \%$ and the mean duration from surgery to recurrence was about 4 years. Endoscopic recurrence rate varies depending on studies; in one study, the rate was $50 \%$ in 3 years, while in another it was $50 \%$ in 5 years after surgery, which were close to our result ${ }^{4,8)}$.

The present study showed that initial intestinal resection, rate of plexitis $<50 \%$, size of the MIG in the myenteric plexus $\geq 867 \mu \mathrm{m}^{2}$, and $\geq 8$ inflammatory cells in the submucosal plexus were associated with postoperative endoscopic recurrence.

Since Ferrante et al. first demonstrated the association between myenteric plexitis and postoperative recurrence ${ }^{14}$, several similar studies have been conducted ${ }^{15-18,24)}$. However, ap- 
Table 3. Univariate Analysis of Pathological Risk Factors Associated with Postoperative Endoscopic Recurrence.

\begin{tabular}{|c|c|c|c|c|c|c|c|c|}
\hline \multirow{2}{*}{ Variable } & \multicolumn{4}{|c|}{ (A) Endoscopic recurrence } & \multicolumn{4}{|c|}{ (B) Endoscopic recurrence in $3 \mathrm{Y}$} \\
\hline & Yes $(n=21)$ & No $(n=19)$ & Cut-off & $P$ & Yes $(n=9)$ & No $(n=19)$ & Cut-off & $P$ \\
\hline Granulomas & $4(19.0)$ & $1(5.3)$ & & 0.188 & $2(22.2)$ & $2(10.5)$ & & 0.408 \\
\hline Inflammatory infiltration of mucosa & $0(0)$ & $3(15.8)$ & & 0.058 & $0(0)$ & $3(15.8)$ & & 0.207 \\
\hline \multicolumn{9}{|l|}{ Number of ganglia } \\
\hline \multicolumn{9}{|l|}{ 1) Submucosal plexus } \\
\hline Number analyzed & $9(42.9)$ & $5(26.3)$ & $\geq 11$ & 0.273 & $6(66.7)$ & $6(31.6)$ & $\geq 11$ & 0.079 \\
\hline Ganglia with plexitis & $6(28.6)$ & $2(10.5)$ & $\geq 9$ & 0.154 & $2(22.2)$ & $0(0)$ & $\geq 19$ & 0.033 \\
\hline Rate of plexitis (\%) & $14(66.7)$ & $15(78.9)$ & $\geq 40$ & 0.385 & $4(44.4)$ & $13(68.4)$ & $\geq 55$ & 0.225 \\
\hline \multicolumn{9}{|l|}{ 2) Myenteric plexus } \\
\hline Number analyzed & $21(100)$ & $17(89.5)$ & $\geq 3$ & 0.127 & $1(11.1)$ & $4(21.1)$ & $\geq 8$ & 0.521 \\
\hline Ganglia with plexitis & $6(28.6)$ & $6(31.6)$ & $\geq 5$ & 0.835 & $2(22.2)$ & $7(36.8)$ & $\geq 5$ & 0.439 \\
\hline Rate of plexitis (\%) & $14(66.7)$ & $16(84.2)$ & $\geq 38$ & 0.200 & $6(66.7)$ & $16(84.2)$ & $\geq 38$ & 0.290 \\
\hline \multicolumn{9}{|l|}{ 1)+2) } \\
\hline Number analyzed & $18(85.7)$ & $12(63.2)$ & $\geq 10$ & 0.099 & $9(100)$ & $12(63.2)$ & $\geq 11$ & 0.035 \\
\hline Ganglia with plexitis & $6(28.6)$ & $3(15.8)$ & $\geq 14$ & 0.333 & $2(22.2)$ & $1(5.3)$ & $\geq 22$ & 0.175 \\
\hline Rate of plexitis (\%) & $10(47.6)$ & $3(15.8)$ & $<50$ & 0.031 & $5(55.6)$ & $16(84.2)$ & $\geq 47$ & 0.102 \\
\hline \multicolumn{9}{|l|}{ Size of MIG } \\
\hline Submucosal plexus $\left(\mu \mathrm{m}^{2}\right)$ & $9(42.9)$ & $4(21.1)$ & $\geq 413$ & 0.141 & $9(100)$ & $18(94.7)$ & $\geq 46$ & 0.483 \\
\hline Myenteric plexus $\left(\mu \mathrm{m}^{2}\right)$ & $18(85.7)$ & $9(47.4)$ & $\geq 867$ & 0.009 & $6(66.7)$ & $3(15.8)$ & $\geq 2966$ & 0.007 \\
\hline \multicolumn{9}{|l|}{ Cellular infiltrates } \\
\hline \multicolumn{9}{|l|}{ 1) Submucosal plexus } \\
\hline Mononuclear cells & $3(14.3)$ & $0(0)$ & $\geq 6$ & 0.086 & $8(88.9)$ & $17(89.5)$ & $\geq 1$ & 0.962 \\
\hline Polymorphonuclear leukocytes & $3(14.3)$ & $0(0)$ & $\geq 3$ & 0.086 & $3(33.3)$ & $0(0)$ & $\geq 3$ & 0.007 \\
\hline Total cells & $4(19.0)$ & $0(0)$ & $\geq 8$ & 0.044 & $9(100)$ & $18(94.7)$ & $\geq 1$ & 0.483 \\
\hline *T-lymphocytes & $4(19.0)$ & $2(10.5)$ & $\geq 2$ & 0.451 & $1(11.1)$ & $1(5.3)$ & $\geq 5$ & 0.574 \\
\hline *Mastocytes & $15(71.4)$ & $9(47.4)$ & $\geq 1$ & 0.120 & $1(11.1)$ & $1(5.3)$ & $\geq 5$ & 0.574 \\
\hline \multicolumn{9}{|l|}{ 2) Myenteric plexus } \\
\hline Mononuclear cells & $8(38.1)$ & $3(15.8)$ & $\geq 5$ & 0.114 & $5(55.6)$ & $7(36.8)$ & $\geq 4$ & 0.350 \\
\hline Polymorphonuclear leukocytes & $2(9.5)$ & $0(0)$ & $\geq 5$ & 0.167 & $2(22.2)$ & $0(0)$ & $\geq 5$ & 0.003 \\
\hline Total cells & $7(33.3)$ & $3(15.8)$ & $\geq 6$ & 0.200 & $3(33.3)$ & $2(10.5)$ & $\geq 9$ & 0.141 \\
\hline *T-lymphocytes & $10(47.6)$ & $10(52.6)$ & $\geq 2$ & 0.751 & $1(11.1)$ & $0(0)$ & $\geq 13$ & 0.139 \\
\hline *Mastocytes & $5(23.8)$ & $1(5.3)$ & $\geq 1$ & 0.100 & $4(44.4)$ & $1(5.3)$ & $\geq 1$ & 0.011 \\
\hline
\end{tabular}

$P$ values were calculated by chi-squared tests.

*T-lymphocytes and mastocytes were detected by immunohistochemistry.

Y: years; MIG: Most severely inflamed ganglion.

Table 4. Univariate Analysis of Endoscopic Recurrence and Dimensions and Inflammatory Infiltrates in the Most Severely Inflamed Ganglion (MIG).

\begin{tabular}{lccc}
\hline \multirow{2}{*}{ Variable } & \multicolumn{2}{c}{ Endoscopic recurrence } & \multirow{2}{*}{ Yes $(\mathrm{n}=21)$} \\
& & No $(\mathrm{n}=19)$ & \\
\hline (1) Submucosal plexus & & & \\
Size of MIG $\left(\mu \mathrm{m}^{2}\right)$ & $597.4 \pm 1172.1$ & $276.9 \pm 247.2$ & 0.284 \\
Number of inflammatory infiltrates/* & $0.86 \pm 0.63$ & $1.20 \pm 0.90$ & 0.233 \\
(2) Myenteric plexus & & & \\
Size of MIG $\left(\mu \mathrm{m}^{2}\right)$ & $1999.4 \pm 1530.3$ & $1433.7 \pm 1438.0$ & 0.136 \\
Number of inflammatory infiltrates/* & $0.28 \pm 0.24$ & $0.35 \pm 0.34$ & 0.694 \\
\hline
\end{tabular}

$P$ values were calculated by non-parametric Wilcoxon tests.

*Number of inflammatory infiltrates in MIG/Size of MIG $\left(\mu \mathrm{m}^{2}\right) \times 100$.

propriate methods of pathological analysis have not been firmly established, and the precise measurements were not mentioned in any of the preceding studies. Therefore, in the current study, certain protocols for microscopic examination of the proximal margins were established. First, the observa- tion area was limited to within $5 \mathrm{~mm}$ from the proximal margin. Nerve bundles and ganglia showing only one neural cell body were also excluded from the definition of plexus. In previous studies, total numbers of inflammatory infiltrates in H\&E-stained slides and on immunostaining (for anti-CD3 
and anti-CD117 antibodies) were counted to assess the severity of plexitis. However, in the present study, numbers for each slide were counted separately because it was noticed that cells observed in the slides were often different due to the thickness $(3 \mu \mathrm{m})$ of each serial section (some cells appeared while others disappeared in consecutive slides). Particular value was placed on the results observed in $\mathrm{H} \& \mathrm{E}$ slides because H\&E staining is the most common staining procedure and is performed routinely. Therefore, using the results of microscopic observation of H\&E slides would be clinically useful.

Even though the pathological examination methods in the present study were not identical to those in preceding studies, multivariate analysis showed that the presence of $\geq 8$ inflammatory cells in the submucosal plexus is an independent risk factor for endoscopic recurrence. This result was similar to that of a study by Misteli et al., which showed that severe myenteric plexitis is a risk factor for surgical recurrence ${ }^{177}$. The results of the second analysis, the outcome of which was endoscopic recurrence within 3 years after surgery, also revealed that $\geq 3$ polymorphonuclear leukocytes in the submucosal plexus, $\geq 5$ polymorphonuclear leukocytes in the myenteric plexus, and $\geq 1$ mastocytes in the myenteric plexus increased the risk of endoscopic recurrence, although these were not significant factors on multivariate analysis. In some previous studies, submucosal mastocytes and eosinophils were associated with an increased risk of postoperative recurrence ${ }^{15,16}$. Degranulation of mastocytes and eosinophils involves the release of inflammatory mediators. Therefore, these cells have been reported to play important roles in the pathogenesis of inflammatory bowel disease. Mastocytes are closely associated with peptidergic nerves in the gut and are related to hyperplastic nerves. They contain mediators such as tryptase, which inactivates vasoactive intestinal peptide. The release of mediators is upregulated during acute inflammation ${ }^{25,26}$. Eosinophils contain granules, the main contents of which are cytotoxic cationic proteins. The release of neurotoxic granular products from eosinophils may explain the alterations in enteric nerve function seen in $\mathrm{CD}^{27}$. Plexitis characterized by infiltration of mastocytes or eosinophils might therefore be associated with inflammation and lead to postoperative recurrence.

The result of the multivariate analysis also showed that the rate of plexitis $<50 \%$ was an independent risk factor for endoscopic recurrence, which was contrary to the previous results. The rate of plexitis was not revealed in the preceding studies. Therefore, we could only speculate that the severity of plexitis in MIG, not the number of ganglia with plexitis, was an important predictive factor of recurrence.

The current study also paid special attention to the size of the MIG. When ganglia were examined microscopically, the size of ganglia was found to vary considerably among patients, particularly in the myenteric plexus. Therefore, the sizes of the MIG in the submucosal and myenteric plexuses were compared between recurrence and non-recurrence groups. Although not significant, the sizes of the MIG in both submucosal and myenteric plexuses were larger in the recurrence group. In $\mathrm{CD}$, transmural inflammation is associated with lesions of enteric nerve system, such as neuronal hypertrophy and hyperplasia, and an irregular increase in number of nerve fibers and ganglia. Therefore, we estimated that the large size of MIG might reflect the severity of inflammation ${ }^{13,14}$. However, the number of cellular infiltrates per unit area of the MIG in both submucosal and myenteric plexuses was smaller in the recurrence group and the difference was more evident in the submucosal plexus. From these findings, we hypothesized that the size of the MIG in the myenteric plexus might be an efficient predictor of postoperative recurrence, while the number of cellular infiltrates in the submucosal plexus could also be a predictor of recurrence. Moreover, the size of the MIG may be a more reliable indicator than the number of cellular infiltrates. This is because the evaluation of plexitis, which requires counting the number of cellular infiltrates, would involve significant inter-observer variability, since evaluation methods, such as the definition of "one plexus" and "inflammatory cells contiguous with a ganglion," have not yet been firmly established. On the other hand, the size of the plexus represents a more objective evaluation parameter that can be easily measured on H\&E slides. Since this is the first study focusing on the size of the MIG as a histopathological predictor of postoperative recurrence of $\mathrm{CD}$, further studies are needed to confirm the accuracy of this finding. Establishment of pathological examination procedures is essential for the precise evaluation of plexitis.

On multivariate analysis, the values of odds ratios were too large to evaluate and some values of the $95 \%$ confidence interval (CI) were undetectable. On the other hand, the results of R-squared and the area under the ROC curve showed high accuracy of the logistic model, which might support the probability of each variable being a risk factor for endoscopic recurrence. However, we were unable to identify meaningful clinical risk factors for postoperative endoscopic recurrence. The only independent clinical risk factor was the lack of previous intestinal resection, which was contrary to the common theory that previous surgery for $\mathrm{CD}$ is a risk factor for recurrence ${ }^{28,29}$. One of the clinical factors that might have influenced this difference in the results of the present and previous studies was postoperative treatment, especially anti-TNF therapy. Of the 40 patients who underwent postoperative colonoscopy, 23 patients had no history of intestinal resection. Twelve $(52.1 \%)$ of these patients were treated with anti-TNF after surgery, of whom 9 (39.1\%) had recurrence. On the other hand, in 17 patients with history of previous intestinal resection, $7(41.1 \%)$ were treated with anti-TNF and $2(11.7 \%)$ of them had recurrence. From these findings, postoperative anti-TNF therapy had little influence on endoscopic recurrence in this study. Other reasons for the difference are considered to be as follows. First, the cohort in the present study was not large enough due to the retrospective, single-center design. This represents a limitation of the study. Second, the study period 
was very long, spanning 18 years. Moreover, timing and indication for postoperative endoscopic evaluation was not fixed and we were unable to determine when exactly recurrences appeared. Furthermore, we could have failed to detect recurrence in asymptomatic cases. Third, significant disparities were seen in continuous variables that did not show normal distributions, which may have impacted the analyses.

In conclusion, initial intestinal resection, rate of plexitis $<50 \%$, submucosal plexitis with $\geq 8$ inflammatory cells, and a myenteric plexus with size $\geq 876 \mu \mathrm{m}^{2}$ in the proximal resection margin were predictive of postoperative endoscopic recurrence in $\mathrm{CD}$. Pathological examination of the proximal resection margin in the surgical specimen may help to estimate the risk of postoperative recurrence. In particular, the size of the MIG in the myenteric plexus and the number of inflammatory cells in the submucosal plexus are likely to be effective biological markers for predicting recurrence of $\mathrm{CD}$ after surgical resection.

\section{Acknowledgement}

The authors are grateful for the statistical support provided by Dr. Satoru Shimizu (Medical Research Institute, Tokyo Women's Medical University) and for the pathology support provided by Dr. Noriyuki Shibata (Department of Pathology 1, Tokyo Women's Medical University) and Dr. Yoji Nagashima (Department of Surgical Pathology, Tokyo Women's Medical University).

\section{Conflicts of Interest}

There are no conflicts of interest.

\section{References}

1. Brzezinski A, Lashner BA. Inflammatory bowel diseases. 3rd ed. New York (United States of America): Churchill Livingstone, 1997. Chapter 54, Natural history of Crohn's disease; p. 475-483.

2. Bouguen G, Peyrin Biroulet L. Surgery for adult Crohn's disease: what is the actual risk? Gut 2011 Sep; 60(9): 1178-1181.

3. Connelly TM, Messaris E. Predictors of recurrence of Crohn's disease after ileocolectomy: a review. World J Gastroenterol 2014 Oct; 20(39): 14393-14406.

4. Bordeianou L, Stein SL, Ho VP, et al. Immediate versus tailored prophylaxis to prevent symptomatic recurrences after surgery for ileocecal Crohn's disease? Surgery 2011 Jan; 149(1): 72-78.

5. Rutgeerts P, Geboes K, Vantrappen G, et al. Natural history of recurrent Crohn's disease at the ileocolonic anastomosis after curative surgery. Gut 1984 Jun; 25(6): 665-672.

6. Fichera A, Lovadina $S$, Rubin $M$, et al. Patterns and operative treatment of recurrent Crohn's disease: a prospective longitudinal study. Surgery 2006 Oct; 140(4): 649-654.

7. Kane SV, Flicker M, Katz-Nelson F. Tobacco use is associated with accelerated clinical recurrence of Crohn's disease after surgically induced remission. J Clin Gastroenterol 2005 Jan; 39(1): 3235.

8. Buisson A, Chevaux JB, Allen PB, et al. Review article: the natural history of postoperative Crohn's disease recurrence. Aliment Pharmacol Ther 2012 Mar; 35(6): 625-633.

9. Van Assche G, Dignass A, Reinisch W, et al.; European Crohn's and Colitis Organisation (ECCO). The second European evidence- based consensus on the diagnosis and management of Crohn's disease: Special situations. J Crohns Colitis 2010 Feb; 4(1): 63-101.

10. Anseline PF, Wlodarczyk J, Murugasu R. Presence of granulomas is associated with recurrence after surgery for Crohn's disease: experience of a surgical unit. Br J Surg 1997 Jan; 84(1): 78-82.

11. Malireddy K, Larson DW, Sandborn WJ, et al. Recurrence and impact of postoperative prophylaxis in laparoscopically treated primary ileocolic Crohn disease. Arch Surg 2010 Jan; 145(1): 42-47.

12. Kotanagi H, Kramer K, Fazio VW, et al. Do microscopic abnormalities at resection margins correlate with increased anastomotic recurrence in Crohn's disease? Retrospective analysis of 100 cases. Dis Colon Rectum 1991 Oct; 34(10): 909-916.

13. Geboes K, Rutgeerts P, Ectors N, et al. Major histocompatibility class II expression on the small intestinal nervous system in Crohn's disease. Gastroenterology 1992 Aug; 103(2): 439-447.

14. Ferrante M, de Hertogh G, Hlavaty T, et al. The value of myenteric plexitis to predict early postoperative Crohn's disease recurrence. Gastroenterology 2006 May; 130(6): 1595-1606.

15. Sokol H, Polin V, Lavergne-Slove A, et al. Plexitis as a predictive factor of early postoperative clinical recurrence in Crohn's disease. Gut 2009 Sep; 58(9): 1218-1225.

16. Bressenot A, Chevaux JB, Williet N, et al. Submucosal plexitis as a predictor of postoperative surgical recurrence in Crohn's disease. Inflamm Bowel Dis 2013 Jul; 19(8): 1654-1661.

17. Misteli H, Koh CE, Wang LM, et al. Myenteric plexitis at the proximal resection margin is a predictive marker for surgical recurrence of ileocaecal Crohn's disease. Colorectal Dis 2015 Apr; 17(4): 304-310.

18. Decousus S, Boucher AL, Joubert J, et al. Myenteric plexitis is a risk factor for endoscopic and clinical postoperative recurrence after ileocolonic resection in Crohn's disease. Dig Liver Dis 2016 Jul; 48(7): 753-758.

19. Satsangi J, Silverberg MS, Vermeire S, et al. The Montreal classification of inflammatory bowel disease: controversies, consensus, and implications. Gut 2006 Jun; 55(6): 749-753.

20. Rutgeerts P, Geboes K, Vantrappen G, et al. Predictability of the postoperative course of Crohn's disease. Gastroenterology 1990 Oct; 99(4): 956-963

21. Bernell O, Lapidus A, Hellers G. Risk factors for surgery and recurrence in 907 patients with primary ileocaecal Crohn's disease. Br J Surg 2000 Dec; 87(12): 1697-1701.

22. De Cruz P, Kamm MA, Hamilton AL, et al. Crohn's disease management after intestinal resection: a randomised trial. Lancet 2015 Apr; 385(9976): 1406-1417.

23. Olaison G, Smedh K, Sjödahl R. Natural course of Crohn's disease after ileocolic resection: endoscopically visualised ileal ulcers preceding symptoms. Gut 1992 Mar; 33(3): 331-335.

24. Ng SC, Lied GA, Kamm MA, et al. Predictive value and clinical significance of myenteric plexitis in Crohn's disease. Inflamm Bowel Dis 2009 Oct; 15(10): 1499-1507.

25. Raithel M, Winterkamp S, Pacurar A, et al. Release of mast cell tryptase from human colorectal mucosa in inflammatory bowel disease. Scand J Gastroenterol 2001 Feb; 36(2): 174-179.

26. Bloom S. Inflammatory bowel diseases. 3rd ed. New York (United States of America): Churchill Livingstone, 1997. Chapter 11, Mast cells and eosinophils in inflammatory bowel disease; p. 87-93.

27. Hällgren R, Colombel JF, Dahl R, et al. Neutrophil and eosinophil involvement of the small bowel in patients with celiac disease and Crohn's disease: studies on the secretion rate and immunohistochemical localization of granulocyte granule constituents. Am J Med 1989 Jan; 86(1): 56-64.

28. McLeod RS, Wolff BG, Ross S, et al. Recurrence of Crohn's dis- 
ease after ileocolic resection is not affected by anastomotic type: results of a multicenter, randomized, controlled trial. Dis Colon Rectum 2009 May; 52(5): 919-927.

29. Vaughn BP, Moss AC. Prevention of post-operative recurrence of Crohn's disease. World J Gastroenterol 2014 Feb; 20(5): 1147-1154.
Journal of the Anus, Rectum and Colon is an Open Access article distributed under the Creative Commons Attribution-NonCommercial-NoDerivatives 4.0 International License. To view the details of this license, please visit (https://creativecommons.org/licenses/by-nc-nd/4.0/). 